\title{
TRACHEAL REIMPLANTATION OF THE RIGHT UPPER LOBE BRONCHUS WITH SLEEVE MAIN AND INTERMEDIUS BRONCHUS RESECTION AND RECONSTRUCTION
}

\author{
Carsten Schröder, MD, Bernd Vogel, MD, Jürgen Krause, MD, and Paolo Macchiarini, MD, PhD, Hannover, Germany
}

\begin{abstract}
Iatrogenic tracheobronchial injuries most often are tears that result from endotracheal intubation, diagnostic or operative endoscopies, long-term ventilatory support or barotraumas, or injury during procedures adjacent to the trachea (eg, mediastinoscopy or esophagectomy). Such tears usually are partial or complete disruptions that are immediately recognized, and thus effective surgical therapy provides excellent functional outcomes. We describe a delayed bronchial sleeve resection of an iatrogenic laceration of the membranous wall of the right bronchial tree with reanastomosis between the middle-lower lobe carina and main bronchus takeoff and reimplantation of the upper lobe bronchus into the distal, ventral, intrathoracic trachea.
\end{abstract}

Clinical summary. A 31-year-old woman presented with recurrent severe hemoptysis after having had coughing and bronchospastic attacks for 1.5 years. Her history revealed that a posttraumatic fracture of the T6 vertebral body had been stabilized 2.5 years previously by means of transpleural place-

From the Department of Thoracic and Vascular Surgery, Heidehaus Hospital, Hannover Medical School, Hannover, Germany.

Received for publication June 21, 2000; accepted for publication July 27, 2000.

Address for reprints: Paolo Macchiarini, MD, PhD, Department of Thoracic and Vascular Surgery, Heidehaus Hospital, Hannover Medical School, Am Leineufer, 70, 30419 Hannover, Germany (E-mail: pmacchiarini@ compuserve.com).

J Thorac Cardiovasc Surg 2001;121:388-90

Copyright (C) 2001 by The American Association for Thoracic Surgery

0022-5223/2001 $\$ 35.00+0 \quad \mathbf{1 2 / 5 4 / 1 1 0 4 6 5}$

doi:10.1067/mtc.2001.110465 ment of 2 self-fixing screws that were connected with a longitudinal bar into the vertebral bodies of T5 and T7. The operation was through a right posterolateral thoracotomy, and the operative report made no mention of any encounters with the bronchial system. However, an early postoperative chest x-ray film and a computed tomographic (CT) scan showed a dislocation of the $\mathrm{T} 5$ screw and proximity between the prosthetic connecting bar and the right main bronchus. She was discharged from the hospital after an apparently smooth postoperative course, and 6 months later, she had resumed full physical activity, including parachuting. Twelve months after the operation, she was readmitted with coughing that was triggered by deep breathing and bronchospastic attacks, which were treated with corticosteroids and $\beta$-mimetic puffers. She had progressive loss of strength that led to physical inactivity. On admission to our intensive care unit, thoracic CT (Fig 1) and examination with a rigid bronchoscope (Fig 2) showed a perforated pars membranacea starting at the carinal origin of the right main bronchus and extending to the distal aspect of the bronchus intermedius. The findings were compatible with damage that had been caused by the proximal screw and the longitudinal bar of the internal fixation prosthesis. There were no clinical signs of active pleural or mediastinal infection, but there was a small infiltrate in the ventral segment of the right upper lobe. Endoscopically, no active bleeding was found, but the CT scan showed that the azygos vein was in close proximity to the head of the T5 screw, and the point of the screw abutted the lateral wall of the descending aorta.

These findings, together with the history of recurrent hemoptysis, were considered an emergency indication to remove the internal fixation prosthesis. An iterative postero- 


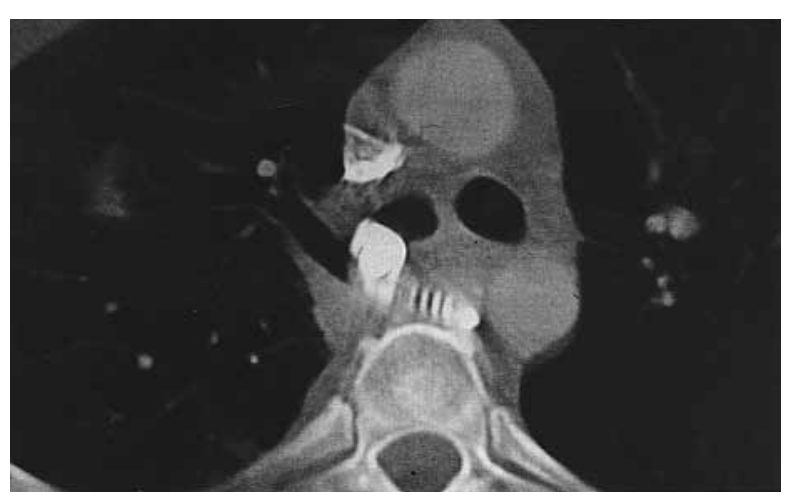

Fig 1. CT scan showing the invagination of the head of the longitudinal bar into the proximal right main bronchus.

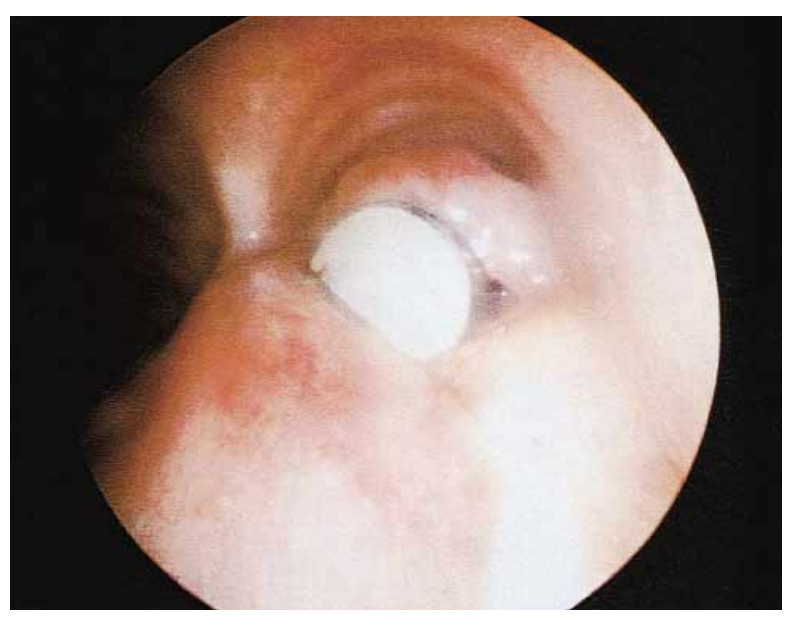

Fig 2. Endoscopic view showing the head of the longitudinal bar invaginated into the membranous wall of the right main bronchus shortly after its origin.

lateral thoracotomy was made, and the pleural space was entered through the fifth intercostal space. The entire dorsal segments of the upper and lower lobes were densely attached to the respective parietal pleura of the costovertebral groove, and the entire transverse apex of the azygos vein cross was covered by fibrotic tissue. Following an extrapleural plane, the azygos vein was ligated proximally and distally to the fibrotic area. Systematic dissection resulted in freeing the right bronchial tree from the lung parenchyma anteriorly and from the midesophagus posteriorly. The entire posterior or membranous wall of the right bronchial tree, from the right main bronchus to the middle-lower lobe carina, had been lacerated and invaginated by the head of the proximal screw and the connecting bar. Care was taken to preserve as much of the peribronchial blood supply as possible. The endobronchial system was then purposely entered by opening the junction of the cartilaginous and membranous bronchus at the origin of the intermedius bronchus. Endoluminal inspection showed a

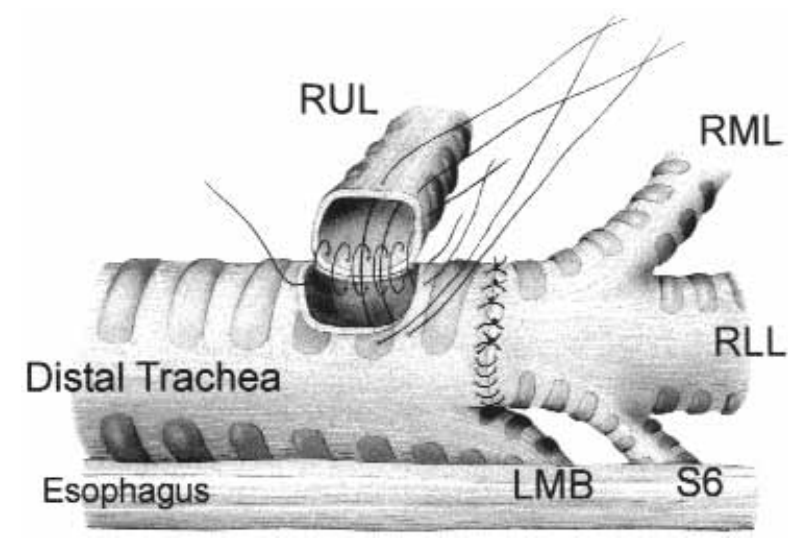

Fig 3. Drawing showing the creation of the $1-\mathrm{cm}^{2}$ oval orifice into the distal trachea, which allowed the reimplantation of the right upper lobe bronchus. This was made after resection of the entire membranous wall of the right bronchial system, reimplantation of the middle-lower lobe carina into the origin of the remnants of the right main bronchus, resection of the upper lobe takeoff, and completion of the posterior part of the great fissure. The view is from a right posterolateral thoracotomy. $R U L$, Right upper lobe; $R M L$, right middle lobe; $R L L$, right lower lobe; $L M B$, left main bronchus; $S 6$, sixth segment of the RLL.

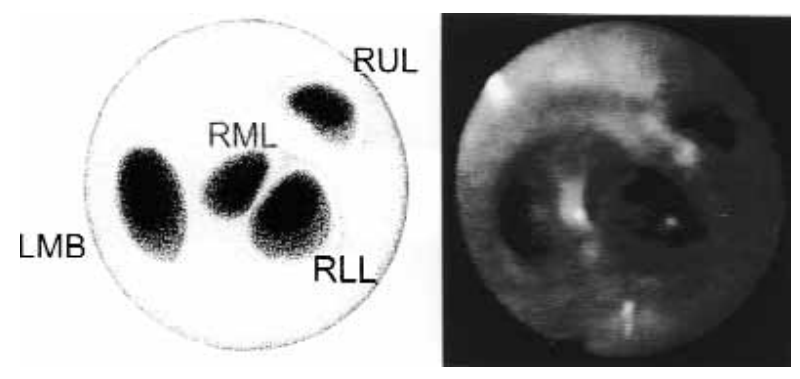

Fig 4. Three months of postoperative endoscopy control, showing patent and well vascularized anastomoses. $R M L$, Right middle lobe; $R L L$, right lower lobe; $L M B$, left main bronchus; $R U L$, right upper lobe.

complete destruction of the pars membranacea, leaving no chance for a primary longitudinal repair.

Given that the takeoffs of the right upper, middle, and lower lobes were intact, the length of the intrathoracic trachea was short, and the bronchial tissues were supple, the decision was made to avoid a pneumonectomy and to undertake a bronchial sleeve resection with reconstruction of the right bronchial tree. The inferior pulmonary ligament was divided and ligated, followed by section of the right main bronchus $0.5 \mathrm{~mm}$ from its origin and the distal intermedius bronchus at the level of the middle-lower lobe carina. The proximal and distal screws and longitudinal bar were then uneventfully 
removed, and the bronchial continuity was reestablished by means of an end-to-end and tension-free anastomosis between the origin of the right main bronchus and the middle-lower lobe carina with a running 4-0 polydioxanone (PDS; Ethicon, Inc, Somerville, NJ) suture on the mediastinal bronchial walls and interrupted 3-0 polyglactin (Vicryl; Ethicon Inc) suture on the anterolateral bronchial walls. The bronchial anastomosis was then circumferentially wrapped with a pedicled pericardial fat flap to avoid erosion of the adjacent pulmonary artery. The remnants of the lacerated membranous and cartilaginous components of the bronchial tree were then removed. The upper lobe bronchus was then sectioned shortly after its takeoff and reimplanted into the ventral distal trachea, where a $1-\mathrm{cm}^{2}$ oval orifice had previously been created (Fig 3) with the same anastomotic technique and material. To avoid tension on this site, the pretracheal fascia was longitudinally opened up to the cervical region. Intraoperatively, after reconstruction, all lobes were fully inflatable. Bronchoscopy showed patent anastomoses, and therefore the endotracheal tube was removed in the operating room. The postoperative course was uneventful, and the patient was discharged on the twelfth postoperative day. Three months after operation, the patient was asymptomatic, and chest x-ray films and bronchoscopy specimens (Fig 4) were normal.

Comments. Isolated laceration of the right main bronchus by the spicula of vertebral body fragments has been described, ${ }^{1}$ but we have found no previous report of a complete laceration of the entire membranous wall of the right bronchial system after spine operations. In our patient it is reasonable to believe that the proximal screw was not firmly fixed and not fully transvertebral and that because of this, the head of the screw eroded progressively into the right main bronchus, carrying with it the longitudinal connecting bar. It seems most likely that the hemoptysis was the result of erosion of the peribronchial arteries, but we cannot exclude the possibility that the bleeding came from the azygos vein. The extent of the laceration made a simple repair impossible. Given the patients' youth, every effort was made to avoid sacrifice of any lung parenchyma. This was possible by respecting the principles of airway reconstruction. The initial tracheobronchotomies at the proximal main right bronchus and at the distal bronchus intermedius allowed assessment of the lesion and resection of the entire lacerated membranous wall. Reconstruction of the right bronchial airway was by an end-to-end anastomosis, according to the method described above, which is usually used for any tracheobronchial reconstruction. ${ }^{2}$ Adequate release of tissues to accomplish tension-free anastomoses was obtained by sectioning the pulmonary ligament and opening the entire pretracheal fascia. We extended the lessons that had been learned from bronchoplastic ${ }^{3}$ and carinal reconstruction ${ }^{4}$ by resecting the upper lobe takeoff along with the lacerated membranous wall and reimplanting the upper lobe bronchus into the ventral distal trachea. Care was taken to respect anatomic relations and the geometry of reconstruction. Indeed, the potential risks of this reimplantation were believed to be reasonable because the intrathoracic trachea was short enough to avoid anastomotic tension. Had this not been the case, tension on the anastomosis could alternatively have been avoided by using an infrahilar Ushaped pericardial release.

Conclusion. We have reported the first bronchial injury after reconstruction of spinal trauma and reimplantation of the right upper lobe bronchus into the distal trachea. The entirely lacerated membranous wall of the right bronchial tree was removed through an extended bronchial sleeve resection, and a pneumonectomy was avoided by reanastomosing the middle-lower lobe carina to the main bronchus takeoff and the upper lobe bronchus to the distal intrathoracic trachea.

\section{REFERENCES}

1. Korovessis PG, Stamatakis M, Baikousis A. Unrecognized laceration of main bronchus caused by fracture of the T6 vertebra. Eur Spine J 1998;7:72-5.

2. Kuhn K, Pasch S, Wojciechowski W, Macchiarini P. Combined sleeve lobectomy and Ivor Lewis esophagectomy for a synchronous primary lung and barret esophagus. J Thorac Cardiovasc Surg 2000;119:1289-90.

3. Wain J. Bronchoplastic procedure. In: Nyhus LM, Baker RJ, Fisher JE, Kaiser L, editors. Cardiothoracic techniques: mastery of cardiothoracic surgery. Philadelphia: Lippencott-Raven; 1998. p. 257-65.

4. Dartevelle P, Macchiarini P. Carinal resection for bronchogenic carcinomas. In: Ross D, Grillo HC, editors. Tracheal surgery. Seminars in thoracic and cardiovascular surgery. Philadelphia: WB Saunders; 1996. p. 414-25. 